\title{
¿Por qué estudiar Sociología?: un análisis de los factores que intervienen en la elección de la Sociología como carrera universitaria en estudiantes de la PUCP
}

\author{
Edith Huerta Gonzales \\ edith.huerta@pucp.edu.pe \\ Pontificia Universidad Católica del Perú (Lima, Perú) \\ (iD) https://orcid.org/0000-0001-6119-6455
}

\author{
Nadia Duffó Chapilliquén \\ a20170835@pucp.edu.pe \\ Pontificia Universidad Católica del Perú (Lima, Perú) \\ (iD) https://orcid.org/0000-0001-8765-5461
}

\begin{abstract}
Resumen
La presente investigación tiene como objetivo explorar los factores principales que influyen en la elección de la carrera en la educación superior universitaria. Se centra particularmente en el caso de lxs ${ }^{*}$ jóvenes estudiantes de Sociología en la Facultad de Ciencias Sociales de la Pontificia Universidad Católica del Perú (PUCP). De esta manera, se busca alimentar el corpus de investigaciones sobre la elección de la carrera profesional, y contribuir a la caracterización de lxs estudiantes y futurxs sociólogxs del país. Para ello, el estudio propone emplear un marco teórico que recoja la teoría de la elección racional, la teoría de reproducción social y la perspectiva de género. La metodología empleada sigue un enfoque mixto aplicado en una muestra no probabilística. Esta combina una encuesta anónima virtual autoaplicada por 43 de lxs estudiantes matriculadxs en el semestre 2020-2 y entrevistas cualitativas a un pequeño grupo de estxs con el fin de profundizar las respuestas sobre su trayectoria educativa y vínculo con la especialidad. Los principales resultados señalan que, en el $76 \%$ de lxs estudiantes de Sociología, la carrera que actualmente estudian no fue la primera opción, sino que iniciaron estudiando otras profesiones. Adicionalmente, se advierte que, en un gran número de casos, la decisión de optar por esta especialidad se enmarca en un entorno institucional, en el cual la influencia de profesores y los cursos relacionados con la disciplina de ciencias sociales son factores relevantes. En cuanto a los motivos, prevalece el valor intrínseco de la carrera sobre el potencial económico de esta. En vista de ello, se prevé la pertinencia e importancia de replicar este tipo de estudios en el resto de las universidades del Perú que ofrecen formación en dicha especialidad.
\end{abstract}

\section{Palabras clave}

Sociología, jóvenes, elección profesional, PUCP, educación superior

\section{(c) (1)}

* En el presente artículo, las autoras han optado por el uso de la "x" como signo neutro para evitar las distinciones por género cuando se amerite. 


\title{
Why study Sociology?: an analysis of factors that intervene in the choice of Sociology as a university career in PUCP students
}

\author{
Edith Huerta Gonzales \\ edith.huerta@pucp.edu.pe \\ Pontificia Universidad Católica del Perú (Lima, Perú) \\ (iD) https://orcid.org/0000-0001-6119-6455

\section{Nadia Duffó Chapilliquén} \\ a20170835@pucp.edu.pe \\ Pontificia Universidad Católica del Perú (Lima, Perú) \\ (iD) https://orcid.org/0000-0001-8765-5461
}

\begin{abstract}
The following research aims to explore the main factors that influence the choice of career in university higher education. It focuses particularly on the case of young Sociology students at the Faculty of Social Sciences of Pontificia Universidad Católica del Perú (PUCP). It seeks to feed the corpus of research on the choice of professional career and contribute to the characterization of students and future sociologists in the country. The study proposes to use a theoretical framework that includes theory of rational choice, theory of social reproduction and gender perspective. The methodology used follows a mixed approach applied in a nonprobabilistic sample. This combines a self-administered virtual anonymous survey by 43 of the students enrolled in the 2020-2 semester and qualitative interviews with a small group of these to deepen the responses about their educational trajectory and link with the specialty. The main results indicate that, in $76 \%$ of Sociology students, the career they are currently studying was not their first option, but they started studying other professions. Additionally, it is noted that, in many cases, the decision to opt for this specialty is framed in an institutional environment, in which the influence of professors and courses related to the discipline of social sciences are relevant factors. Regarding the motives, the intrinsic value of the career prevails over its economic potential. In view of this, the relevance and importance of replicating this type of study in the rest of Peruvian universities that offer training in this specialty is foreseen.
\end{abstract}

\section{Keywords}

Sociology, youth, professional choice, PUCP, higher education 


\section{Introducción}

La elección de la carrera universitaria se presenta como una de las decisiones más importantes en la vida de lxs jóvenes (Guerra y Quevedo, 2007) tras terminar la educación secundaria y determinar si, efectivamente, se desea cursar estudios superiores universitarios o técnicos, iniciar la trayectoria laboral, o llevar a cabo ambas actividades a la par. En algunos casos más que en otros, la situación individual del joven y de su familia puede enmarcar estas decisiones en un contexto de mayor oportunidad o libertad de elección debido a factores económicos, culturales, entre otros. En el caso del Perú, en 2019, la tasa de transición de lxs graduadxs de secundaria que tuvieron oportunidad e iniciaron estudios superiores fue de $36.6 \%$ (ESCALE, 2020).

Los estudios de pregrado suelen marcar el inicio de la trayectoria académica o laboral, que presumiblemente definirá gran parte de las actividades, círculos sociales y fuentes de futuros ingresos de lxs estudiantes. Para cierto grupo de jóvenes, los estudios superiores pueden servir como un mecanismo efectivo para favorecer la movilidad social (Benavides, 2004). Las expectativas de lxs jóvenes con respecto a estas cuestiones suelen ser indicadas como algunos de los factores que orientan la elección de la carrera. Asimismo, se presentan otros aspectos que inciden en dicha deliberación -ya sean de influencia más directa o indirecta- como las motivaciones personales, la autopercepción de habilidades, el género, etc. Desde diferentes disciplinas y con diferentes propósitos, se ha buscado investigar y dar respuesta a la pregunta de cómo lxs estudiantes del nivel superior eligen la carrera que seguirán.

La psicología suele ubicar la discusión en el marco del desarrollo personal de lxs jóvenes y de su identidad. Los estudios psicológicos sobre la elección de la carrera se interesan por la construcción de la orientación vocacional, y los procesos de evaluación de las motivaciones personales y las capacidades autopercibidas. Por otra parte, diferentes disciplinas, desde las mismas facultades y casas de estudio a las que pertenecen, se proponen averiguar los factores que atraen a sus estudiantes a las carreras específicas que ofrecen. Tal es el caso de estudios realizados sobre la elección de carreras en salud y en pedagogía, y el cuaderno de trabajo presentado a la especialidad de Sociología de la PUCP sobre el perfil y expectativas de lxs estudiantes que optan por cursar esta carrera (Zárate et al., 2020; Bobadilla, 2018). La perspectiva sociológica, por su parte, propone una mirada sobre factores estructurales y supraindividuales. Así, cuestiones como la clase socioeconómica, la trayectoria escolar, las redes sociales, etc., contribuyen a un ensayo más completo de los procesos de deliberación y elección realizados por lxs estudiantes.

Específicamente en el caso de carreras liberales o humanistas, diferentes autores identifican tendencias de lxs estudiantes hacia una mayor valoración de cualidades intelectuales o altruistas que pueden ser trazadas en el entorno familiar y diferenciadas por género (Mullen, 2014; Quadlin, 2020). En el marco de las investigaciones sobre la elección de lxs estudiantes por carreras en ciencias sociales, preguntarnos por el proceso de decisión de estudiantes de Sociología en la Pontificia Universidad Católica de la Perú (PUCP) permite conocer mejor los perfiles, orientaciones y aspiraciones de futurxs sociólogxs peruanxs de nuestra facultad. 
La presente investigación propone una exploración actualizada que aporte a la discusión general sobre la elección de la carrera bajo la guía de la siguiente pregunta principal: ¿qué factores contribuyen a la elección de la carrera universitaria para lxs estudiantes del pregrado de Sociología en la PUCP? De este modo, se intenta complementar aquel cuaderno de trabajo con información reciente y con un énfasis en factores relacionados con la crianza y el entorno familiar de procedencia de lxs jóvenes. Para ello, se plantean las siguientes preguntas secundarias: ¿cómo dan cuenta lxs estudiantes de su elección de estudiar Sociología en la PUCP?, ¿cuál fue el rol del entorno familiar y otros entornos previos en la elección de la carrera de lxs jóvenes? y ¿cómo se vincula la elección de la carrera de lxs estudiantes con sus expectativas y aspiraciones laborales?

\section{Enfoque teórico}

En esta sección, presentaremos tres de las principales teorías y enfoques que explican, desde sus aproximaciones, la decisión de la carrera profesional. La primera se basa en la teoría de acción racional, en la cual el estudiante elige una carrera de acuerdo con las expectativas del mercado y las oportunidades disponibles. La segunda teoría gira en torno a la reproducción social. Esta propone que, de acuerdo con el modelo de crianza y las características del hogar (e.g. el ingreso económico, relación con la cultura y el sistema educativo), el joven se atribuye ciertas capacidades, habilidades e intereses que influyen en la elección de la carrera. Por su parte, la perspectiva de género complementa lo mencionado al señalar que las mujeres tienden a experimentar esta elección de forma desigual. Finalmente, se presenta el modelo de marco teórico en el que se enmarca la investigación.

\subsection{Teoría de elección racional}

Entender la definición de la carrera por seguir en los estudios superiores como una elección invita a enmarcar la discusión bajo la teoría de la elección racional. De esta manera, se busca entender cómo lxs jóvenes deciden sus trayectorias de vida de acuerdo con sus intereses personales y oportunidades, y a partir de la examinación de la información disponible. Este enfoque proveniente de la economía propone, en términos generales, analizar las decisiones, elecciones y acciones de lxs actores sociales como racionales. En este, "se elige deliberadamente el mejor medio disponible para alcanzar una meta dada, que se persigue también deliberadamente" (Cristiano, 2006, p. 138), y dicha meta "surge de un conjunto de preferencias ordenadas [...], transitivas [...], y constantes en el tiempo" (ibid). Si bien este postulado es ampliamente criticado por expresar criterios que pocas veces se cumplen a cabalidad empíricamente, Javier Cristiano (2006) defiende su uso en investigaciones sociológicas por un principio de utilidad metodológica y en consideración de varios aportes rectificatorios que admiten cierta irracionalidad o, más bien, una aplicación de racionalidad imperfecta o compleja.

Tal como se expone anteriormente, la elección de la carrera puede ser atribuida a una diversidad de factores que abarcan desde cuestiones que se interpretan como preferencias personales hasta factores sociales y estructurales, y que operan de formas más 
o menos perceptibles sobre el actor deliberante. Asumir conscientemente la "presunción razonable" de que lxs actores resuelven sus decisiones racionalmente permite partir de la noción general de que la elección de carrera puede seguir estrategias maximizadoras de beneficios, sin pretender explicar -y más bien admitiendo- otros referentes teóricos para indagar sobre las categorías que clasifican y definen las preferencias que cada uno maneja (Cristiano, 2006).

Los criterios más cercanos a la noción de racionalidad aplicados en la toma de decisión de la carrera universitaria se presentan, tal vez, en aquellos vinculados a las expectativas y aspiraciones laborales y remunerativas en el futuro. Estos factores más pragmáticos contemplan cuestiones como el abanico de oportunidades de trabajo, y de movilidad horizontal o vertical (en términos de la diversidad de posiciones y posibilidades de ascenso) que provee el mercado laboral. Es así como parte de esta deliberación puede basarse en lecturas e interpretaciones de la estructura real del mercado laboral apoyada en información limitada e incompleta, e incluso muchas veces imprecisa. Asimismo, las aspiraciones laborales de lxs jóvenes actores pueden verse orientadas, en mayor medida, por valoraciones no económicas, como motivaciones altruistas o intrínsecas, tal como identifica Mullen (2014) para el caso de estudiantes universitarixs de carreras liberales.

En esa línea, la teoría relativa de aversión al riesgo (relative risk aversion) -propuesta de Brenn y Goldthrope (1997) - sugiere que lxs padres y madres pueden tener una influencia en la elección de la carrera de sus hijxs sobre la base de un criterio similar a la búsqueda maximizadora de beneficios. Se trata específicamente de una búsqueda orientada a velar por la seguridad de lxs hijxs en tanto mantengan o mejoren la posición social de ventaja en la cual iniciaron su trayectoria. En ese sentido, la elección de la carrera es importante, dado que esta elección implica algún riesgo en cuanto a las oportunidades de trabajo y las recompensas financieras (Brenn y Goldthorpe, 1997). Padres y madres, dentro de su análisis propio del mercado, pueden influenciar o limitar las decisiones de lxs hijxs de optar por carreras que ellxs consideran "riesgosas" y, en vez de ello, optar por carreras con grandes expectativas económicas. Estas influencias o consideraciones podrían aparecer, por ejemplo, en el caso de lxs estudiantes de la PUCP de estratos bajos que tienden a estudiar carreras pensando en el retorno y estabilidad económica (Bobadilla, 2018).

Aproximarnos a la elección racional de la carrera supone la deliberación racional del individuo en una serie de condiciones. Al respecto, resulta importante mencionar que la "racionalidad" es construida por el entorno cercano. Esta puede ser diferente de acuerdo, por ejemplo, con la clase y el contexto social en los cuales se desenvuelven y socializan lxs jóvenes. El documento de trabajo elaborado por Bobadilla (2018) sobre lxs estudiantes de la unidad de Estudios Generales Letras ${ }^{1}$ y de la carrera de Sociología en la PUCP sugiere que las escalas de pago de lxs estudiantes guardan relación con la importancia que estxs otorgan a la remuneración y al grupo de carreras que eligieron (Bobadilla, 2018). En esa

1 El paso por la unidad académica de Estudios Generales Letras es obligatorio para todxs lxs estudiantes de la PUCP que pertenecen a carreras profesionales de Ciencias Sociales, Humanidades, Gestión y Alta Dirección, Comunicación, Contabilidad, Derecho, entre otras. El objetivo de llevar un período de formación en dicho ámbito es aportar a la formación integral, fortalecer los intereses vocacionales y la constitución ética y ciudadana a través del aprendizaje multidisciplinario con perspectiva humanista (EE. GG. LL., 2020). 
línea, la composición diferenciada de los criterios de racionalidad permite que la decisión óptima no siempre esté medida en la misma proporción por los mismos factores.

\subsection{Teoría de la reproducción social}

Si bien planteamos la elección de la carrera como un acto de deliberación -que, en sentido más estricto, lo es-, la perspectiva de la elección racional nos permite e invita a indagar también cómo se construyen los intereses, capacidades y juicios de valor que últimamente guían dicha decisión. La teoría de la reproducción social, a partir del desarrollo teórico de Pierre Bourdieu (1973) en el estudio de la educación, ofrece un marco de comprensión para la relación entre origen social y el devenir de la trayectoria educativa y laboral que vale la pena examinar. Esta parte de una aproximación para el análisis del sistema educativo entendido como una institución no neutral que admite y reproduce desigualdades entre estudiantes de diferentes clases sociales, que terminan definiendo los intereses, habilidades y desempeño de lxs estudiantes en su trayectoria educativa y académica.

En primer lugar, este enfoque se centra en cómo el nivel socioeconómico familiar reproduce las ventajas adquiridas en lxs hijxs. Esto influye en el acceso a la educación y su calidad, ya que lxs hijxs de las familias más acomodadas tienden a ir a mejores colegios y suelen tener acceso a una educación de mayor calidad. En esa línea, la relación del medio familiar con la cultura transmitida en el universo escolar repercute en una adquisición desigual entre alumnos y alumnas de habilidades, intereses y conocimientos premiados por el sistema educativo (Bourdieu, 1973). Además, el autor explica que el sistema educativo recompensa las habilidades de las clases altas, por lo cual hay una ventaja de lxs alumnxs dentro del sistema educativo por su predisposición.

Las investigaciones de Lareau (2015), por ejemplo, sobre los modelos de crianza entre familias de clase baja y alta, encuentran diferencias sustanciales al respecto. Por un lado, las familias de clase alta ponen en práctica un "cultivo concertado" (concerted cultivation), una lógica cultural de crianza a través de la cual inculcan talentos y habilidades a sus hijxs, lo que facilita el éxito escolar del alumnx; además, inculcan valores como la independencia y su capacidad de participación. En cambio, las familias de clase baja tienen un modelo de "crianza de crecimiento natural" (accomplishment of natural growth), en el que lxs padres dejan crecer a sus hijxs libremente y les permiten relacionarse con su grupo de pares. Además, priorizan enseñarles valores como el respeto y la obediencia, especialmente a la autoridad.

En ese sentido, las preferencias de lxs padres por ciertas habilidades y el involucramiento en la educación de sus hijxs se ven reflejados en los intereses y preferencias que lxs menores desarrollan (Ma, 2009). Así, si el padre no tiene interés por las matemáticas, posiblemente no las enseñe a su hijx; en cambio, si le gusta pintar, es probable que le enseñe esta habilidad o influya cierta apreciación del arte. En relación con esto, la exposición de una habilidad al hijo incrementa la posibilidad de dominarla y sentir interés por ella, lo cual puede conducir a elegir estudiar una carrera relacionada.

En cuanto al ámbito formativo, Bourdieu (1973) señala que los entornos educativos son espacios de reproducción cultural/social en los que se refuerzan inadvertidamente ciertas capacidades y habilidades de la clase dominante. Las habilidades de lxs hijxs de una 
familia acomodada son recompensadas en el colegio mediante las calificaciones, lo cual refuerza el interés, la autopercepción de capacidades y la confianza en sí mismxs. Por el contrario, si lxs padres no se involucran en la educación de lxs hijxs o carecen de recursos culturales similares a los requeridos por el sistema educativo, es probable que su influencia sea irrelevante en la formación de sus intereses.

Además del entorno familiar, otros entornos previos a la etapa de la elección de la carrera pueden alentar ciertas preferencias y reafirmar evaluaciones que el joven contempla. Los espacios educativos en los que participa durante la trayectoria escolar, por ejemplo, pueden ser determinantes en virtud de su rol socializador e introductorio al ámbito académico. Ello se produce mediante el autorreconocimiento de las capacidades individuales, que puede, a su vez, alimentar un interés sobre cierto tipo de práctica académica y profesional (Pérez y Vergara, 2002), la transmisión tácita de nociones y valoraciones sobre las carreras, así como la exposición al relacionamiento con contactos y referentes académicos. El desempeño escolar, como señala Bourdieu (1973), vincula en gran medida la familia de procedencia con la cultura escolar, así como las enseñanzas explícitas e implícitas en diferentes instituciones educativas. Entonces, a partir de lo señalado, las características familiares influyen en la creación y reforzamiento de capacidades, valores y habilidades que son premiados en la escuela. Así, estos factores contribuyen a entender cómo se construyen las preferencias vocacionales que inciden en la deliberación por la elección de la carrera.

\subsection{Perspectiva de género}

En el nivel de las características personales que inciden en la decisión de la carrera, es posible distinguir también cómo factores estructurales y sociales influyen en la construcción de orientaciones y motivaciones. Ello se puede identificar en las lógicas de elección de la carrera en jóvenes estudiantes diferenciadas por género. Esta es una variable transversal dentro del análisis, dado que los roles de género se construyen y refuerzan en los diferentes espacios sociales, como la familia, las redes sociales, el entorno educativo, entre otros. Investigaciones como las de Ma (2009), Navarro y Casero (2012), y Quadlin (2020) señalan una tendencia en las mujeres a tomar la decisión de la carrera por estudiar según el valor intrínseco de la profesión, es decir, por el interés que genera la carrera (en lo intelectual o en su valoración altruista) o las proyecciones propias, como la orientación vocacional. En cambio, las decisiones de los hombres suelen verse orientadas, en mayor medida, por las recompensas externas de la carrera, como la retribución económica o el prestigio.

Retrocediendo a etapas previas en la trayectoria vital, la experiencia de niños y niñas en el sistema educativo, así como otros espacios sociales, se manifiesta de forma diferenciada. Las niñas, especialmente, pueden encontrar ciertas desventajas al ser orientadas a relacionarse con la escuela, a usar juguetes y a tener expectativas de vida sobre la base de roles de género tradicionales que pueden generar un desinterés por las habilidades o materias vinculadas a atributos masculinos. Rodríguez (2008), por ejemplo, da cuenta de las situaciones de discriminación que afectan a un grupo de mujeres que estudian o ejercen carreras de Ingeniería, entendidas como un campo profesional masculinizado. En 
muchos casos, se evidencia también el rechazo de sus familias y cuestionamientos a su expresión de género u orientación sexual, lo cual puede desalentar la decisión de estudiar estas especialidades o desempeñarse en ellas.

Por otro lado, los estudios de segregación horizontal analizan la división social del trabajo, en función del género, que existe dentro de los campos profesionales. Es decir, se abocan a observar la composición de hombres y mujeres dentro de un mismo oficio o ámbito profesional. Según Navarro y Casero (2012), esta mantiene patrones provenientes de los roles de género de la sociedad, lo que explica la tendencia de que, en comparación con los hombres, se encuentre mayormente mujeres en carreras asociadas a las tareas de cuidado por vocación e interés en ayudar a otras personas. Al respecto, Mullen (2014) presta atención a los procesos de elección de la carrera condicionados por el género en hombres, quienes construyen sus identidades en oposición de la feminidad. Debido a esto, suelen tomar distancia de los programas de estudio y materias vinculadas con atributos femeninos, y orientan sus estudios a líneas de carrera que les permitan mantener el estatus social o ascender económicamente de cara al rol proveedor que anticipan asumir cuando formen su propia familia.

A partir de la bibliografía revisada sobre diferentes aproximaciones hacia el problema de investigación y la construcción del marco teórico, es posible señalar algunos factores principales que influyen en la deliberación por la elección de la carrera universitaria. A modo de resumen, se presenta un diagrama sobre las relaciones entre las variables de mayor incidencia.

Gráfico 1. Diagrama resumen del enfoque teórico

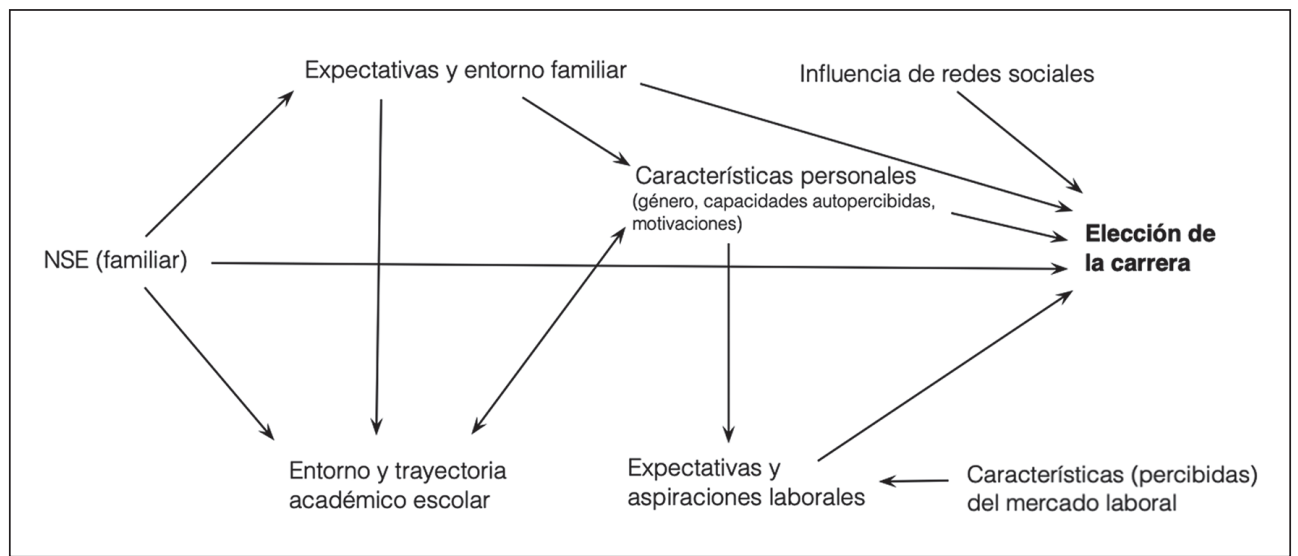

Elaboración propia. Gráfico inspirado en el modelo sociopsicológico del logro de estatus de Wisconsin. (Hauser, Tsai y Sewell, 1983)

El enfoque de la teoría de la elección racional ofrece un marco en el cual situar y analizar las lógicas de elección de lxs jóvenes. Junto con la teoría de la reproducción social, la elección de la carrera se construye a lo largo de sus trayectorias de vida a partir de las motivaciones y aspiraciones. El nivel socioeconómico familiar mantiene una contribución en el modelo de crianza y la transferencia de habilidades/conocimientos 
que se reforzarán en los espacios educativos. Sobre la base de lo anterior, se plantea que lxs jóvenes eligen carreras vinculadas a sus habilidades (autopercibidas), así como a las orientaciones y motivaciones que han desarrollado en los entornos mencionados. Además, otros factores como el género y las redes sociales pueden marcar una diferencia en torno a las motivaciones y a la elección de una carrera.

Metodología

La metodología empleada en esta investigación es mixta, dada la combinación de métodos cuantitativos y cualitativos. Por un lado, se elaboró una encuesta con el fin de conocer el rol del entorno familiar, las expectativas y aspiraciones laborales dentro del proceso de elección de la carrera de Sociología. Dicha encuesta, autoaplicada y de carácter anónimo, fue realizada durante la segunda mitad de 2020 mediante el software Google Forms, y se difundió por medio de plataformas de redes sociales vinculadas a lxs estudiantes de Sociología de la PUCP.

En el semestre 2020-2, se encontraban matriculadxs 114 estudiantes de Sociología en la Facultad de Ciencias Sociales PUCP (información extraída a partir de la consulta del Campus virtual PUCP). La muestra fue aleatoria no probabilística, y el cuestionario fue respondido por 43 estudiantes de Sociología en la facultad de Ciencias Sociales ${ }^{2}$. Se ha optado por no contemplar a lxs estudiantes de Estudios Generales Letras (EE. GG. LL.), ya que se considera que la elección por la carrera en esta población estudiantil no se encuentra - generalmente- tan consolidada (en comparación con el grupo de estudio).

La encuesta fue aplicada del 8 al 15 de diciembre de 2020. Previamente, se realizó una prueba piloto con cuatro participantes. La mayoría de las respuestas provino de estudiantes mujeres (73\%), y los hombres representaron el $27 \%$ de lxs encuestadxs. Las edades fluctuaron entre 19 y 21 ańos. La mayoría de las respuestas provino de estudiantes de sexto $(25.6 \%)$ y octavo $(37.2 \%)$ ciclo que se dedican exclusivamente a estudiar. El nivel socioeconómico familiar de lxs estudiantes se proyectó a partir de la escala de pagos en la que se encontraban. Estas escalas económicas son evaluadas y asignadas por la Universidad a partir de un análisis de la capacidad de pago de las familias mediante el balance económico que presenta cada una. Para ello, se toman en cuenta los ingresos, egresos, fuentes de ingreso, el patrimonio, entre otras variables (DAES, 2020). Las escalas y los montos se clasificaban en cinco diferentes escalas hasta 2016; sin embargo, fueron modificadas a nueve escalas a partir de 2017. Por ello, en la Universidad, coexisten dos escalas de pago diferenciadas que se aplican de acuerdo con el año de ingreso. A continuación, presentamos la escala de pagos vigente en la PUCP de 2020 a 2021.

2 La mayoría de los planes de estudios de las carreras que ofrece la PUCP implica pasar por las facultades de Estudios Generales Letras o Ciencias los primeros cuatro ciclos. En el caso de la especialidad de Sociología, esta comprende diez ciclos y dos etapas, la primera en EE. GG. LL y la segunda en la Facultad de Ciencias Sociales, que comienza en el quinto hasta el décimo ciclo de estudios. Para mayor información, revisar el siguiente enlace: https://facultad. pucp.edu.pe/ciencias-sociales/carreras/sociologia/plan-de-estudios/ 
Tabla 1. Escalas de pago y derecho de matrícula expresados en soles peruanos según el año del ingreso, 2020

\begin{tabular}{|c|c|c|c|c|c|}
\hline \multicolumn{3}{|c|}{ Escala de pago a partir de 2017} & \multicolumn{3}{|c|}{ Escala de pago hasta 2016} \\
\hline Escala de pago & Monto por crédito & $\begin{array}{l}\text { Derecho de } \\
\text { matrícula }\end{array}$ & Escala de pago & Monto por crédito & $\begin{array}{l}\text { Derecho de } \\
\text { matrícula }\end{array}$ \\
\hline G1 & $S / 263.8$ & S/ 108.3 & 1 & S/ 297.7 & S/ 55.7 \\
\hline $\mathrm{G} 2$ & $S / 338.3$ & S/ 136.5 & 2 & $S / 390.4$ & $S / 70.8$ \\
\hline G3 & S/ 424.4 & $S / 169.3$ & 3 & $\mathrm{~S} / 520.2$ & $S / 91.4$ \\
\hline G4 & $S / 520.2$ & S/ 205.7 & 4 & S/ 692.1 & $\mathrm{~S} / 118.5$ \\
\hline G5 & $S / 623.2$ & S/ 244.8 & 5 & S/ 931.3 & S/ 157.6 \\
\hline G6 & $S / 729.2$ & $S / 285.1$ & & & \\
\hline G7 & S/ 832.9 & $S / 324.5$ & & & \\
\hline G8 & $\mathrm{S} / 928.2$ & $S / 360.7$ & & & \\
\hline G9 & $S / 1008.2$ & $S / 391.2$ & & & \\
\hline
\end{tabular}

Fuente: Portal del Estudiante PUCP. Elaboración propia.

En esa línea, el $30 \%$ de lxs estudiantes de Sociología pertenecen a un nivel socioeconómico alto (escalas 4-5, y G6, G7, G8 y G9)3 . Por otro lado, se realizaron cuatro entrevistas semiestructuradas con un enfoque narrativo a dos hombres y dos mujeres estudiantes de Sociología de la PUCP con fin de conocer el proceso de elección de la carrera. Estxs no habían elegido la carrera de Sociología como su primera opción y tenían una trayectoria académica particular, por lo cual se decidió entrevistarlxs. La identidad de cada estudiante se mantiene en reserva y, en este artículo, se emplea seudónimos asignados por las autoras para referirse a ellxs.

Tabla 2. Descripción de estudiantes de Sociología participantes en las entrevistas a profundidad

\begin{tabular}{|l|l|}
\hline Entrevistadxs & \multicolumn{1}{c|}{ Descripción } \\
\hline José & $\begin{array}{l}\text { Cursa el octavo ciclo. Tiene } 23 \text { años. Estudió Medicina y luego Psicología Social en dos universidades } \\
\text { diferentes; sin embargo, las carreras no se alineaban con sus intereses. Tuvo un tutor sociólogo que lo } \\
\text { introdujo a la sociología y lo incentivó a estudiar en la PUCP. Actualmente, se encuentra trabajando en } \\
\text { consultoras. }\end{array}$ \\
\hline Mayte & $\begin{array}{l}\text { Cursa el octavo ciclo. Tiene } 24 \text { años. Estudió Administración hasta el octavo ciclo en otra universidad. } \\
\text { Entonces, llevó un curso de Género, mostró mayor interés por el tema y decidió su cambio de carrera a la } \\
\text { PUCP. }\end{array}$ \\
\hline Muriel & $\begin{array}{l}\text { Cursa el noveno ciclo. Tiene 22 años. Desde su egreso del colegio se vio interesada por estudiar Sociología; } \\
\text { sin embargo, aspectos relacionados al "perfil" del sociólogo la desanimaron. Tras inscribirse a los programas } \\
\text { de pregrado de Historia y Antropología, finalmente, se trasladó a Sociología. }\end{array}$ \\
\hline Ernesto & $\begin{array}{l}\text { Cursa el octavo ciclo. Tiene 23 años. Ingresó a la PUCP con la intención de estudiar la carrera de Derecho. Se } \\
\text { trasladó a otras dos universidades y, posteriormente, volvió a la PUCP, donde inició la carrera de Sociología. }\end{array}$ \\
\hline
\end{tabular}

Fuente: Trabajo de campo propio, 2020. Elaboración propia.

3 A modo de ejemplo, lxs alumnxs de escala G9 pueden llegar a pagar 4110 soles mensualmente durante cinco meses, y el total de ciclo sería S/ 20 555, si es que llevan 20 créditos. 
El enfoque de esta investigación es un estudio de caso a partir del cual se busca conocer las particularidades del grupo, y se centra en pocos casos para un análisis exhaustivo (Della Porta, 2013). La PUCP, fundada en 1917, es la primera universidad privada del país. Actualmente es considerada una de las más prestigiosas y, en Latinoamérica, se posiciona en el puesto 15 del listado ${ }^{4}$ de mejores universidades. Este reconocimiento se debe a su alta reputación académica y de lxs empleadores (PuntoEdu, 2020). A pesar de las escalas diferenciadas, se considera una de las universidades más costosas del país. En cuanto a las carreras que ofrece la PUCP, es la única universidad privada con la especialidad de Sociología. Lxs estudiantes de dicha especialidad en esta universidad solo representan el $7.3 \%$ del total de matriculados de esta especialidad en el país (Sunedu, 2017).

En este estudio de caso, nos enfocaremos en un grupo de jóvenes que optaron por estudiar Sociología en la única universidad privada que ofrece la especialidad y que tiene un costo mensual por encima del salario mínimo, lo cual implica una posición económica favorable en comparación con otrxs estudiantes de la misma carrera en otras universidades. $\mathrm{Al}$ presentar este tipo de características, buscamos entender cómo lxs estudiantes de esta especialidad optan por elegir Sociología frente a otras carreras.

La primera forma del análisis de la información se centra en los resultados recogidos en las encuestas realizadas de forma virtual. Por ello, se realiza análisis de datos y se elabora tablas según características que pueden ser diferenciadas en ciertos grupos, como el caso del género. La segunda forma de análisis se realiza a partir de la información recogida en las entrevistas, que es transcrita y procesada de acuerdo con las principales dimensiones de la investigación. Su análisis va de la mano con los datos señalados, dado que esta información complementa y contextualiza lo recogido.

\section{Resultados}

A continuación, se exponen los principales resultados, divididos según los objetivos planteados en el diseño de investigación. Primero, se presentan cuestiones generales sobre cómo lxs estudiantes se acercaron a la Sociología y decidieron estudiar la carrera; luego, las aspiraciones y expectativas laborales que pudieron intervenir en su proceso de deliberación; y, finalmente, el rol de los entornos (principalmente el familiar) -previos al ingreso a la carrera- en la construcción de preferencias y en el proceso de elección. Asimismo, los resultados contemplan el género de las personas participantes como una variable que tomar en cuenta de forma transversal.

\subsection{Elección de la carrera}

Observando la trayectoria educativa de lxs estudiantes participantes de la investigación, se destaca una considerable proporción de aquellxs que no iniciaron los estudios superiores con la intención de dirigirse a Sociología. El 76.7 \% de lxs estudiantes -en proporciones similares para hombres y mujeres- optó por trasladarse de especialidad e incluso de universidad una vez iniciados sus estudios universitarios. Los resultados recogen, además,

$4 \quad$ Ranking Quacquarelli Symonds (QS) Mundial 2021 
que la decisión por cambiar de carrera se presentó principalmente dentro de la PUCP -en un $60.5 \%$ de la muestra-, ya sea en el paso por la unidad de Estudios Generales Letras $(51.2 \%)$ o en otras facultades de pregrado (9.3\%). Otro grupo de encuestadxs, que representa el $9.3 \%$, tomó la decisión de estudiar Sociología mientras se encontraba en otra universidad, y un $27.9 \%$, en etapas previas a los estudios superiores.

Tabla 3. Porcentaje de estudiantes según la etapa en la que tomaron la decisión de estudiar Sociología, por género

\begin{tabular}{|l|c|c|c|}
\hline Etapa en que tomó la decisión de estudiar Sociología & Total & Femenino & Masculino \\
\hline Total & 100 & 100 & 100 \\
\hline En la infancia & 2.3 & 0.0 & 7.1 \\
\hline En el colegio & 11.6 & 10.7 & 7.3 \\
\hline Entre el colegio y la universidad & 14.0 & 17.9 & 5.1 \\
\hline En Estudios Generales Letras & 51.2 & 50.0 & 7.1 \\
\hline En la universidad (en alguna facultad de la PUCP) & 9.3 & 10.7 & 14.3 \\
\hline En la universidad (no la PUCP) & 9.3 & 7.1 & 0.0 \\
\hline Otra & 2.3 & 3.6 & \\
\hline
\end{tabular}

Fuente: Trabajo de campo propio, 2020. Elaboración propia.

Las entrevistas cualitativas permiten esclarecer lo intrincados o complejos que pueden llegar a ser los procesos de definición de la carrera para algunxs estudiantes. Estos pueden tomar idas y venidas, así como implicar varios cambios en la vida de lxs jóvenes. El caso de Ernesto destaca por su ingreso inicial a la carrera de Derecho en la PUCP, su posterior traslado a otras dos universidades y su retorno a la primera institución para estudiar el pregrado de Sociología. A partir de su experiencia, cuenta lo siguiente: "Llegué a la Sociología casi sin proponérmelo. [...] No sabia nada de Sociología. Absolutamente, creo que nada. Nunca se me hubiera pasado por la mente". No fue hasta un curso de investigación en Estudios Generales Letras que Ernesto conoció la disciplina y decidió seguir investigando sobre esta por sus propios medios. En ese sentido, si bien el ambiente académico institucional permitió cierto acercamiento primario a la carrera que posteriormente eligió, el estudiante identifica que, a través de su iniciativa individual, logró profundizar su entendimiento y tomar finalmente la decisión de cambiar de especialidad.

Mayte y José, por su parte, comparten las experiencias de haber iniciado sus trayectorias en los estudios superiores en otras universidades y, posteriormente, haber ingresado a la PUCP. La primera entrevistada indica que, en el momento de elegir dónde estudiar tras egresar de la secundaria, centró su decisión en una universidad específica antes de evaluar a qué se quería dedicar. A pesar de haber recibido la sugerencia de estudiar Sociología, se opuso a la idea de estudiar dicha carrera y en la PUCP debido a preconcepciones sobre la posición política de la universidad que le desagradaban. Antes de estudiar Sociología, José tenía claro que deseaba "contribuir a la sociedad para bien" trabajando en favor del cierre de las brechas de pobreza en el país. En vista de ello, reconoció la importancia de 
entender la realidad social y vio necesario cambiar sus estudios en Psicología Social por la carrera de Sociología, influenciado por un tutor — sociólogo- que su padre le presentó.

Muriel, por otro lado, se ha mantenido en la misma universidad, pero cambió de especialidad dos veces antes de decidirse por la Sociología. Si bien procuró informarse sobre esta mientras evaluaba qué quería estudiar, en un primer momento, rechazó la idea de postular a este programa de pregrado, pues no consideraba contar con el perfil de estudiante que anunciaba el portal de la universidad.

Los cuatro testimonios recogidos manifiestan la búsqueda de una carrera que podría atender los intereses o expectativas de lxs entrevistadxs por conocer sobre la realidad social e incidir en ella. Si bien la carrera de Sociología en la PUCP define su orientación hacia el estudio de los grupos y estructuras sociales, y el profundo conocimiento de la realidad (PUCP, 2021), su quehacer se enmarca en premisas, métodos y ámbitos de influencia particulares que pueden influir en la percepción que tienen lxs jóvenes sobre la disciplina y, por ende, en el hecho de elegir o no seguir esta carrera. En los casos citados, los factores que alejaron en un primer momento a lxs estudiantes de Sociología de su especialidad incluyen el hecho de no contar con suficiente información sobre la carrera, tener prejuicios sobre esta (no alineados a sus intereses), la priorización de otros factores al momento de tomar la decisión de la carrera y la percepción de las capacidades propias.

En relación con lo anterior, el cuestionario aplicado preguntó a lxs estudiantes de Sociología sobre su interés en los temas sociales y la etapa en la cual identifican el inicio de su orientación hacia ellos. Se encontró que, para la mayoría (65.1\%), este comenzó en las etapas previas a los estudios superiores (la infancia, el colegio, y la etapa entre el colegio y la universidad), mientras que, para el $34.9 \%$, este inició mientras se cursaba la universidad. De este modo, para la muestra de estudiantes, manifestar interés en temas sociales durante la etapa previa a los estudios universitarios no siempre se tradujo en la elección de la especialidad de Sociología. El abordaje de las diversas cuestiones y problemáticas sociales no se limita a esta disciplina, y se presume que, en varios casos, lxs estudiantes habrían optado por otras carreras para ello o habrían postergado estos intereses frente a otros. Aun así, si bien la mayoría de lxs encuestadxs decidieron ingresar a Sociología tras una primera deliberación y luego de explorar otras disciplinas, ello no determina necesariamente que el interés por la especialidad se haya formado de manera tardía.

El desarrollo del interés por la Sociología o por el quehacer sociológico puede ser entendido como alentado o fomentado por entornos de socialización, contactos y referentes variados, ya sea de forma directa o indirecta a lo largo de la trayectoria educativa. Para $30.2 \%$ de lxs estudiantes encuestadxs, el acercamiento a la Sociología se dio por medio de un curso de Estudios Generales Letras y, para un $23.3 \%$, por influencia de docentes 5 . En otros casos, $(37.2 \%)$ también fue importante la revisión y lectura de

5 Cabe aclarar que el cuestionario aplicado no distingue entre docentes de diferentes niveles educativos, por lo cual lxs encuestadxs pueden referirse a contactos docentes de la escuela básica o los estudios superiores indistintamente. 
textos e investigaciones sociológicos ${ }^{6}$. Los resultados del cuestionario y las entrevistas aplicadas sugieren que, si bien el interés por temas sociales pudo surgir previamente, para el acercamiento a la Sociología, en muchos casos, fue importante contar con un contexto institucional educativo que alentase su desarrollo (e.g. las clases en las facultades de estudios generales o en facultades de otras universidades, la relación con docentes o referentes cercanos a la Sociología en la etapa universitaria, temas abordados en cursos, entre otros).

Tabla 4. Porcentaje de encuestadxs según cómo se dio su acercamiento a la Sociología, por género

\begin{tabular}{|l|c|c|c|}
\hline Pregunta*: ¿Cómo se produjo su acercamiento a la Sociología? & Total & Femenino & Masculino \\
\hline Por influencia de amistades & 14.0 & 17.9 & 7.1 \\
\hline Por influencia de familiares & 11.6 & 17.9 & 0.0 \\
\hline Por influencia de docentes & 23.3 & 21.4 & 28.6 \\
\hline Por influencia de vínculos sexoafectivos & 7.0 & 3.6 & 7.1 \\
\hline Por un curso de Estudios Generales Letras & 30.2 & 28.6 & 35.7 \\
\hline Por un curso en el colegio & 2.3 & 0.0 & 7.1 \\
\hline Por revisión y lectura de textos/investigaciones sociológicos & 37.2 & 39.3 & 35.7 \\
\hline Por actividad política & 18.6 & 17.9 & 21.4 \\
\hline Por Internet/medios de comunicación & 11.6 & 10.7 & 14.3 \\
\hline Por ferias vocacionales/plataformas de orientación vocacional & 14.0 & 17.9 & 7.1 \\
\hline Otro & 9.3 & 7.1 & 14.3 \\
\hline
\end{tabular}

*La pregunta mostrada en la tabla admite múltiples respuestas, por lo que los resultados no suman $100 \%$.

Fuente: Trabajo de campo propio, 2020. Elaboración propia.

Sobre la base de lo anterior, se advierte que la decisión por estudiar Sociología suele ser más consecuencia de un prolongado proceso de deliberación antes que una elección rápida o poco examinada. Si bien elegir la carrera por estudiar en el nivel de estudios superiores presupone la necesidad de una sesuda reflexión, el caso de varixs estudiantes de la muestra destaca por haberse decidido por la Sociología incluso luego de haber iniciado sus estudios universitarios en otras especialidades. En un considerable número de casos, el acercamiento inicial a la disciplina se produjo en los primeros cursos en la universidad. Así también, los referentes dentro del contexto educativo -como lxs docentes- ocupan un lugar importante. Otros factores que considerar mencionados con recurrencia son la revisión de literatura académica -que puede darse por cuenta propia o como parte de asignaciones educativas- y la participación política. Estos recuerdan que, si bien factores del contexto (e.g. las redes sociales o el entorno educativo) pueden ser importantes en la construcción de motivaciones, aspiraciones o expectativas, la exploración personal

6 La revisión de literatura sociológica puede haber sido por cuenta propia, o como parte de los requerimientos e indicaciones de docentes o cursos a lo largo de la trayectoria educativa. 
y capacidad de decisión propia son fundamentales y terminan manifestándose en la elección efectiva de la carrera.

\subsection{Aspiraciones y expectativas laborales}

Los resultados referidos a las preguntas sobre aspiraciones y expectativas laborales de lxs estudiantes encuestados sugieren hallazgos similares a los encontrados por el II Censo Nacional Universitario 2010 (INEI, 2010) y a partir de la revisión bibliográfica sobre facultades de ciencias sociales en otros países. En estas investigaciones, la situación económica y la empleabilidad son factores menos importantes en la elección de la carrera frente al resto de opciones vinculadas con las motivaciones personales, las aptitudes e influencias reconocidas de personas en el entorno. Específicamente en el censo de 2010, el $49.9 \%$ de lxs estudiantes de las carreras de Sociología, Antropología, Arqueología, Historia y otras afines indicaron como aspectos principales que influyeron en su elección la orientación vocacional; el $26.8 \%$, sus aptitudes; y, en menor medida, el $9 \%$, la influencia de padres, familiares, docentes y amigos.

Gráfico 2. Aspecto principal que influyó para elegir la carrera (datos del II Censo Nacional Universitario 2010)

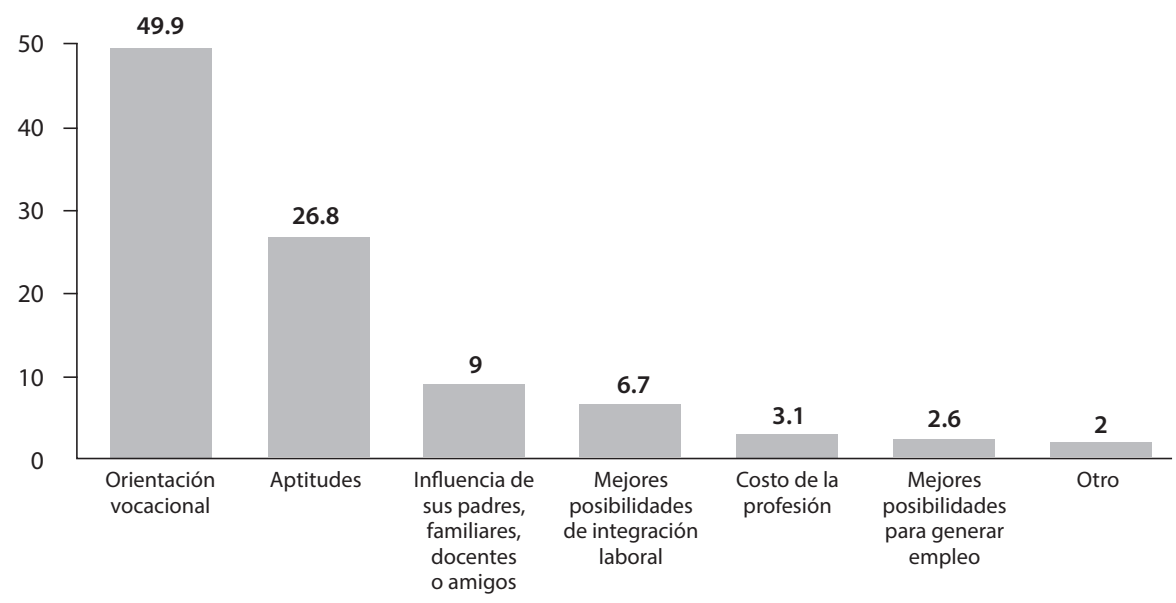

Fuente: II Censo Nacional Universitario (INEI, 2010). Elaboración propia.

En la encuesta aplicada para la presente investigación, el $60.5 \%$ de estudiantes indican la proyección social -entendida como el vínculo entre las aspiraciones personales y el impacto social sobre una comunidad-y las convicciones personales como los factores principales que influyeron en su decisión de carrera, seguido del $46.5 \%$ de respuestas para el interés por ayudar a mejorar la vida de otras personas, y un 32.6\% para el interés en el campo académico. Las opciones propuestas en la encuesta referidas a aspectos económicos y de inserción laboral no fueron indicadas por ningún alumno ni alumna como factores principales. El reconocimiento de aptitudes y habilidades propias afines a la carrera (16.3\%), y la influencia de padres, familiares, docentes y amigxs (7\%) también fueron factores señalados, aunque en menor medida, por algunas de las personas encuestadas. 
Tabla 5. Porcentaje de estudiantes según los factores principales que influyeron en su decisión de carrera, por género

\begin{tabular}{|l|c|c|c|}
\hline Factores principales* & Total & Femenino & Masculino \\
\hline Interés en el campo académico & 32.6 & 25.0 & 50.0 \\
\hline Proyección social y convicciones personales & 60.5 & 75.0 & 35.7 \\
\hline Interés por ayudar a mejorar la vida de otras personas & 46.5 & 57.1 & 28.6 \\
\hline Mis aptitudes/habilidades & 16.3 & 10.7 & 28.6 \\
\hline Mejores posibilidades de integración laboral & 0.0 & 0.0 & 0.0 \\
\hline Mejores posibilidades de asegurar estabilidad económica & 0.0 & 0.0 & 0.0 \\
\hline Influencia de sus padres, familiares, docentes o amigxs & 7.0 & 3.6 & 14.3 \\
\hline Otro & 9.3 & 3.6 & 14.3 \\
\hline
\end{tabular}

*La pregunta mostrada en la tabla admite múltiples respuestas, por lo que los resultados no suman $100 \%$.

Fuente: Trabajo de campo propio, 2020. Elaboración propia.

Con respecto a las respuestas diferenciadas por género, la proyección social y convicciones personales $(75 \%)$ y la posibilidad de ayudar a otras personas $(57.1 \%)$ son los factores mayormente considerados por las mujeres, mientras que, en el caso de los hombres, el interés en el campo académico es la opción más seleccionada (en este caso, por el $50 \%$ de encuestados). Adicionalmente, se distingue una mayor consideración de las habilidades autopercibidas por los hombres que por las mujeres para la elección de la carrera (28.6\% de encuestados frente a $10.7 \%$ de encuestadas), así como una mayor influencia de padres, familiares, docentes o amigxs (14.3\% de encuestados frente a 3.6 $\%$ de encuestadas).

En cuanto a las características más valoradas sobre la carrera de Sociología, encontramos que, entre las personas encuestadas, el $89 \%$ considera muy importante o importante que esta sea una carrera con compromiso social y que contribuya al desarrollo del país. También se valora que permita aportar a la investigación y la discusión académica (77 $\%$ indica que es importante o muy importante), y que tenga un amplio abanico de áreas laborales en las que desempeñarse (75\% lo considera importante o muy importante). En cambio, lxs estudiantes encuestadxs consideran, en menor proporción, que la carrera sea prestigiosa (35\%) o que permita trabajar en cargos públicos de alto nivel (46\%). Aun así, el porcentaje de respuestas muestra que se trata de aspectos igualmente señalados como de gran importancia para algunas personas.

Lo más importante de esta carrera para el conjunto de entrevistadxs, según lo manifestado, sigue siendo el compromiso social, las posibilidades que brinda para contribuir a la comunidad académica, como también en el ámbito social (i.e. en proyectos sociales, y en el activismo político antes que en altos cargos políticos burocráticos), y la variedad de posibilidades en las que se puede desempeńar el o la profesional. Lo primero coincide con una valoración del carácter intrínseco de la carrera en tanto implica un compromiso entre esta, el estudiante y la sociedad.

La carrera de Sociología es percibida por lxs estudiantes como una carrera con proyección social que involucra el conocimiento y el bienestar de un grupo humano. 
Gráfico 3. Nivel de importancia sobre la carrera de Sociología, 2020

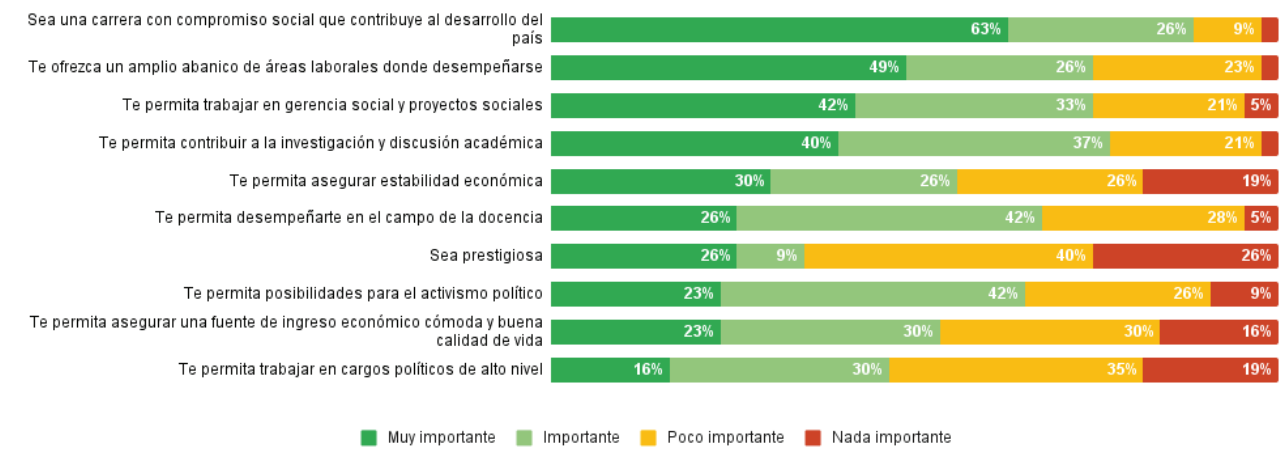

Fuente: Trabajo de campo propio, 2020. Elaboración propia.

Tanto hombres como mujeres tienen un acercamiento e interés por la especialidad que se puede leer de forma diferenciada a través de los sistemas de género. Las mujeres, por ejemplo, orientan su interés por la carrera en torno a valores sociales y habilidades blandas; en cambio, los hombres se interesan por el conocimiento y la academia. La elección de la carrera también es una representación y expresión de las diferencias en género en cuanto a expectativas e intereses de lxs estudiantes.

\subsection{Entorno familiar}

En la dimensión del entorno familiar y la influencia de contactos, se preguntó por el nivel educativo del padre y la madre. Se encontró que lxs padres del $72 \%$ de lxs estudiantes habían culminado una carrera profesional y algunxs incluso contaban con un posgrado. Por otro lado, solo el 62.8 \% de las madres compartía este grado académico. En ese sentido, se presenta una proporción considerable tanto de padres como de madres con niveles de educación superior universitaria completa. La muestra de la encuesta está constituida principalmente por hijxs de profesionales.

A partir de estas cifras, se puede sugerir cierta influencia del nivel educativo familiar en las habilidades, intereses o motivaciones educativas o profesionales de lxs estudiantes. A modo de ejemplo, el testimonio de José presenta una distinción interesante entre la influencia percibida de sus padres, ambxs profesionales. Su mamá, contadora, es considerada "muy técnica y aburrida"; en cambio, su papá, periodista, lo incitaba a leer y discutir sobre temas políticos. Asimismo, el entrevistado cuenta que la experiencia de los viajes por motivos laborales de sus padres jugó un rol importante en el desarrollo de su interés por los temas rurales. Así, si bien el alto grado educativo de lxs padres suele vincularse a un mayor acercamiento a carreras académicas, tomar en cuenta intereses y tipo de profesión puede indicar diferencias importantes.

Por otro lado, al preguntar a lxs estudiantes por el apoyo que recibieron de su entorno familiar respecto de su decisión de estudiar Sociología, solo el $23.3 \%$ ha señalado que recibió apoyo. En cambio, 72 \% de encuestadxs señala que el apoyo ha sido relativo (es decir, no se dio plenamente). Este apoyo relativo consiste en que lxs padres cuestionen 
la decisión de sus hijxs, pero que continúen financiando sus estudios. Al respecto, cabe la posibilidad de que, con el tiempo, lxs padres cambien de opinión a medida que se informen más sobre la carrera y/o avancen los ciclos de estudio, como el caso de José y Muriel. En esa línea, al preguntar por los motivos de la falta de apoyo, la mayoría señala que esta se debe a la preocupación por la estabilidad económica (50 \% de los casos).

Gráfico 4. Motivos de lxs padres para no apoyar la decisión de estudiar Sociología, 2020

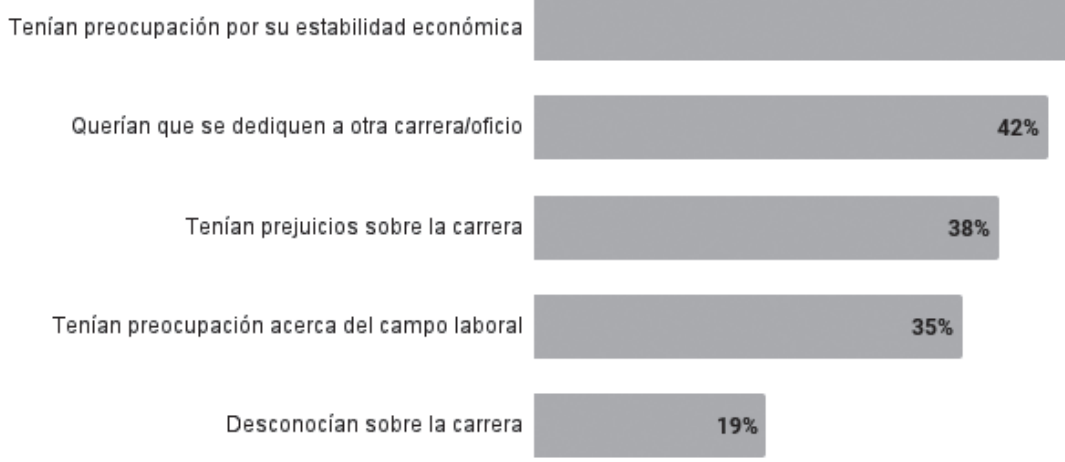

Fuente: Trabajo de Campo propio 2020. Elaboración propia.

Otro aspecto que considerar es el vínculo del estudiante con alguna persona que estudia o haya estudiado carreras de ciencias sociales o humanidades. El 48 \% señaló que sí conoce a alguien dentro de su entorno cercano, ya sean amigos, familia, vínculos afectivos, entre otros. Más aún, para el $58.3 \%$ de ellxs, esta persona influenció en su decisión de estudiar Sociología. En ese sentido, este tipo de influencia directa tiene un impacto importante al tomar la decisión. En las entrevistas, algunxs participantes señalan a algún profesor de Ciencias Sociales como referente que llegó a inspirarlxs: "Quería ser como ép" (José); "quería tener su vida, quiero hacer lo que ella hace" (Mayte), lo cual generó una importante expectativa en torno a estudiar Sociología. Las figuras académicas que conocen lxs estudiantes son importantes, ya que valoran el trabajo que realizan. Además, lxs estudiantes, al visualizar un proyecto de vida concreto y realizado, ven la posibilidad de seguir una carrera similar.

\section{Discusión}

Los principales factores que toman en cuenta lxs estudiantes de Sociología de la PUCP para optar por esta especialidad se centran mayormente en un interés intrínseco de la carrera, puesto que les permite desarrollar sus intereses académicos y contribuir a la sociedad. Adicionalmente, la decisión por estudiar Sociología surge, en su mayoría, de un proceso de deliberación una vez iniciada la etapa universitaria. Al respecto, los cursos y el material académico vinculados a esta carrera resultan elementos y espacios importantes para despertar o alimentar el interés en la especialidad. Asimismo, figuras como profesores o tutores pueden materializar la potencialidad de carrera de poder estudiar aquello que les gusta y poder vivir de ello. 
Bobadilla (2018) señala el vínculo entre las orientaciones profesionales y la escala económica de lxs alumnxs en la PUCP. En su estudio, lxs estudiantes de mayores escalas tienden a restarle importancia al potencial económico de la carrera y a darle mayor valor al conocimiento y al prestigio, a diferencia de lxs estudiantes de menores escalas, quienes tienden a considerar más el tema económico y la proyección profesional. Si bien en nuestra muestra encontramos un $32 \%$ de alumnxs de escalas 4, 5 y G6, G7, G8, G9 -mientras el porcentaje de la investigación anterior es del $50 \%{ }^{7}$-, se encontró que la mayoría de estudiantes de Sociología encuestadxs valora el potencial social de la especialidad por sobre su potencial de retribución económica. Esto se añade al dato sobre el nivel socioeconómico familiar, una considerable proporción de encuestadxs tiene también padres profesionales.

A la luz de lo anterior, la teoría de la aversión al riesgo presume una mayor preocupación por parte de lxs padres sobre la elección de la carrera de lxs hijxs con base en la retribución económica que puedan obtener. Los resultados de la investigación sugieren que, si bien dicha preocupación puede estar presente, esta no se manifiesta de forma impositiva en lxs estudiantes, sino, más bien, en la forma de apoyo relativo. Además, la respuesta de lxs padres con respecto a la decisión de su hijx por la especialidad depende del grado de conocimiento y cercanía sobre la investigación y el rol de sociólogx. Este hallazgo permite considerar el contexto institucional -como las clases y su paso por Estudios Generales Letras- y la relación del entorno familiar con el sistema educativo como factores intervinientes en dicha percepción de riesgo. Cursar los estudios de la carrera de Sociología en una universidad privada, así como la cercanía de algunxs padres y madres al ámbito académico, podría, en cierta medida, disipar los miedos respecto de la especialidad, en tanto se logre identificar un potencial académico y laboral importante para lxs hijxs.

Adicionalmente, aunque lxs estudiantes reconocen cierta influencia del entorno familiar y otros contactos en las redes sociales, el entorno educativo recibe mayor crédito para el caso específico del acercamiento a la Sociología. Si bien muchxs de lxs jóvenes indican haber recibido orientaciones hacia temas sociales antes de ingresar a la universidad, muchxs también declaran que su elección por la carrera se debió a factores del contexto institucional, como haber llevado algún curso o la influencia de docentes. Recordando el hecho de que la gran mayoría de estudiantes inició en otras carreras y luego se trasladó a Sociología, queda claro que, en la práctica, las decisiones que toman lxs actores sociales admiten revisiones y cambios, y que el contexto institucional juega un rol importante en la elección de la profesión.

Uno de los aspectos más interesantes acerca del estudio de lxs estudiantes de Sociología en la PUCP es el alto porcentaje de estudiantes mujeres y las diferencias entre sus motivaciones y aquellas de los estudiantes varones. Las carreras orientadas al cuidado del prójimo son mayoritariamente femeninas, dados los roles de género según los cuales las mujeres, desde pequeñas, se encargan del cuidado y soporte familiar (Navarro y Casero,

7 El autor considera las escalas de pensión de la PUCP que van del 1 al 5. No considera la actualización de escalas a partir de 2017. 
2012). En este caso, las mujeres estudian Sociología por la proyección social y por mejorar las condiciones de vida de lxs demás, factores menos relevantes para los hombres (35\% y $38 \%$, respectivamente), lo cual indica la feminización de la especialidad de Sociología, orientada a mejorar las condiciones de vida de diferentes comunidades. En cambio, los hombres se muestran más interesados en la especialidad por la producción académica.

Por último, tal como Mullen (2014) sugiere, una mayor profundización de la mirada sobre este último aspecto es necesaria para cuestionar las conceptualizaciones estrictamente individuales. Ello habilitaría, además, un análisis mejor contextualizado sobre las diferentes preferencias y orientaciones según el género. Al respecto, consideramos que, aunque la presente investigación provee pistas interesantes sobre cómo las motivaciones en una misma carrera se ven diferenciadas por el género (las mujeres declaran mayores intereses altruistas y de ayudar a lxs demás), investigaciones posteriores pueden brindar un mejor alcance sobre el tema.

\section{Conclusiones}

La presente investigación se interesa por determinar los factores principales que intervienen en la elección de la carrera de estudiantes de Sociología de la PUCP. Se rescatan, principalmente, las consideraciones de lxs jóvenes en el momento de su deliberación, así como diversos aspectos que han influenciado la construcción de preferencias y orientaciones a lo largo de sus trayectorias de vida. Para nuestro caso de estudio, la elección de la carrera en lxs estudiantes se atribuye como iniciativa y deliberación personal antes que como producto de otras influencias. Sin embargo, se logra identificar la importancia del contexto institucional, y, en menor medida, del entorno familiar y las redes sociales para fomentar el interés y reflexión de la elección de la carrera profesional.

Aunque las lógicas metodológicas y analíticas de la teoría de la elección racional permiten el desarrollo de estas reflexiones, la menor importancia sobre las expectativas económicas halladas en la muestra de encuestadxs no debe ser solamente cuestionada desde el argumento de que no es una preocupación urgente. Para ello, es importante la crítica al manejo superficial de esta propuesta e ir más allá de la mirada economicista, preguntando adicionalmente cómo se construyen las orientaciones y preferencias que tomaron en cuenta lxs estudiantes en su elección.

A modo de cierre, para próximos estudios, nos parecería interesante comparar las motivaciones de lxs estudiantes de Sociología de la PUCP y las de lxs estudiantes de otras universidades de Lima que ofrecen esta carrera profesional (e.g. la UNMSM o la UNFV) con el objetivo de analizar la relevancia de la variable económica en los motivos de elección de la carrera. Asimismo, proponemos los estudios sobre el soporte que pueden dar lxs padres a sus hijxs a la luz de sus propias trayectorias educativas, dado que consideramos este un importante factor en estudiantes de universidades privadas. 


\section{Referencias bibliográficas}

Benavides, M. (2004). Educación y estructura social en el Perú: un estudio acerca del acceso a la educación superior y la movilidad intergeneracional en una muestra de trabajadores urbanos. En P. McLauchlan de Arregui, M. Benavides y S. Cueto (Eds.), ¿Es posible mejorar la educación peruana?: evidencias y posibilidades (pp. 125-146). GRADE.

Bobadilla, P. (2018). ¿... y cómo eliges tu profesión? Motivaciones y factores que influyen en la elección de una carrera profesional en estudiantes de Estudios Generales Letras y Sociología. Cuaderno de trabajo. Lima: Departamento de Ciencias Sociales, PUCP. Bourdieu, P. (1973). Cultural reproduction and social reproduction. En R. Brown (Ed.). Knowledge, education, and cultural change. Londres: Tavistock.

Breen, R. y Goldthorpe, J. (1997). Explaining Educational Differentials: Towards a Formal Rational Action Theory. Rationality and Society, 9(3), 275-305.

Cristiano, J. (2006). Racionalidad de la acción y racionalidad de la teoría. Revista Española de Investigaciones Sociológicas, 113(6), 135-153.

Della Porta, D. y Keating, M. (Eds.) (2013). Enfoques y metodologías de las Ciencias Sociales: una perspectiva pluralista. Madrid: Akal.

Estudios Generales Letras. (2020). Misión y visiones. Obtenido de https://facultad.pucp. edu.pe/generales-letras/sobre-eeggll/mision-y-vision/

ESCALE. (s.f.). Tasa de transición, la educación superior (\% de egresados del nivel educativo anterior). Sitio web de la Unidad de Estadística del Ministerio de Educación del Perú http://escale.minedu.gob.pe/ueetendencias2016?p_auth=s0Ocnq1y\&p_p_id= TendenciasActualPortlet2016_WAR_tendencias2016portlet_INSTANCE_t6xG\& p_p_lifecycle $=1 \&$ p_p_state $=$ normal $\&$ p_p_mode $=$ view $\&$ p_p_col_id $=$ column $-1 \&$ p_p_col_pos=1\&p_p_col_count=3\&_TendenciasActualPortlet2016_WAR_ tendencias2016portlet_INSTANCE_t6xG_idCuadro $=111$

Guerra, L. y Quevedo, T. (2007). La elección profesional: momento de particular importancia para el desarrollo personal. Psicología para América Latina. Obtenido de http://pepsic.bvsalud.org/scielo.php?script=sci_arttext\&pid=S1870-350X2007000 300007\&lng=pt\&tlng=es

Hauser, R., Tsai, S.L. y Sewell, W. (1983). A model of stratification with response error in social and psychological variables. Sociology of Education, 56, 20-46. Obtenido de https://www.ssc.wisc.edu/wlsresearch/publications/files/public/Hauser-Tsai-Sewell_ Model.Stratification.R.E.S.P.V.pdf

INEI. (2010). II Censo Nacional Universitario 2010.

Lareau, A. (2015). Invisible Inequality: Social Class and Childrearing in Black Families and White Families. En R. Arum, I. Beattie y K. Ford (Eds.), The structure of schooling: readings in the sociology of education (Tercera edición). Los Ángeles: SAGE Publications.

Ma, Y. (2009). Family Socioeconomic Status, Parental Involvement, and College Major Choices. Gender, Race/Ethnic, and Nativity Patterns. Sociological Perspectives, 52(2), 211-234. 
Mullen, A. (2014). Gender Social Background, and the Choice of College Majors in Liberal Arts Context. Gender and Society, 28(2), 289-312.

Navarro, C. y Casero, A. (2012). Análisis de las diferencias de género en la elección de estudios universitarios. Estudios sobre educación, 22, 115-132.

Pérez, R. y Vergara, A. (2002). Estudio exploratorio sobre los factores que inciden en la elección de la carrera de Sociología en la UAM-Azcapotzalco. Universidad Autónoma Metropolitana. Sociológica, 49(17), 303-352.

Pontificia Universidad Católica del Perú. (2020). Información económica. Obtenido de https://estudiante.pucp.edu.pe/informacion-economica/escala-derechos-academicosy-valor-del-credito/semestre-2020-i/

PuntoEdu. (2020). Ranking QS Mundial 2021: PUCP alcanza su mejor posición histórica entre universidades latinoamericanas. Obtenido de https://puntoedu.pucp.edu.pe/ orgullo-pucp/ranking-qs-mundial-2021-pucp-alcanza-su-mejor-posicion-entreuniversidades-latinoamericanas/

Quadlin, N. (2020). From Major Preferences to Major Choices: Gender and Logics of Major Choice. Sociology of Education, 93(2), 91-109.

Rodríguez, A. (2008). "Aquí hay que hacerse respetar". Mujeres, entre tuercas y metales Una mirada desde las estudiantes de las facultades de Ingeniería de la Pontificia Universidad Católica del Perú. En M. Benavides (Ed.). Análisis de programas, procesos y resultados educativos en el Perú. Contribuciones empiricas para el debate (pp. 177-207). Lima: GRADE. Obtenido de http://www.grade.org.pe/upload/publicaciones/archivo/ download/pubs/LIBROGRADE_ANALISISDEPROGRAMASEDUCATIVOS.pdf SUNEDU. (2017). Estadísticas por universidades por programa de estudios.

Zárate, N., Rocha, I., Vargas, V., González, J. y Ruíz-Xicotencalt, J. (2020). Estudiar Medicina: ¿vocación o moda? Revista de Educación en Ciencias de la Salud, 17(1), 37-40. Obtenido de http://www2.udec.cl/ofem/recs/anteriores/vol1712020/artinv17120f.pdf 